\title{
Prioritäten bei der Therapie des Multiplen Myeloms: Eine Analyse der Perspektive von Ärzten
}

\author{
Axel C. Mühlbacher • Matthias Nübling
}

Online publiziert: 11. März 2014

(C) The Author(s) 2014. Dieser Artikel ist auf Springerlink.com mit Open Access verfügbar

\begin{abstract}
Background: Currently there is no cure for patients with Multiple Myeloma. Hence the primary objective of treatment is to extend survival time at the best possible quality of life. A study for eliciting patient preferences in multiple myeloma was conducted in summer/autumn of 2007. Target audiences have been the patients themselves. In the present study the questionnaire for preferences was presented to physicians, asking about their opinion as to what patients might consider most important. The aim of this second preference survey was to determine the physicians' notions of their patients' preference patterns, and to find out whether they were consistent with the patients' actual preferences.

Study Design (Including Statistical Design): The study consisted of two parts: a pre-test as a qualitative preliminary survey aimed at establishing relevant parameters, and a quantitative principal study with the objective of determining patients' preferences as seen from the perspective of physicians as decision-makers. This part includes a direct assessment for levels of importance in a total of 17 aspects (5-point Likert scale) and a Discrete-choice experiment for
\end{abstract}

A.C. Mühlbacher ( $\varangle)$

Institut Gesundheitsökonomie und Medizinmanagement (IGM),

Hochschule Neubrandenburg, Brodaer Straße 2,

17033 Neubrandenburg, Deutschland

e-mail: muehlbacher@hs-nb.de

A.C. Mühlbacher

Duke Global Health Institute, Duke University, Durham, NC, USA

A.C. Mühlbacher · M. Nübling

Gesellschaft für Empirische Beratung GEB mbH, Hauptstrasse

67.1, 79211 Denzlingen, Deutschland

M. Nübling

e-mail: nuebling@empirische-beratung.de measuring preferences, consisting of eight pairs with eight characteristics each.

Sample \& Methods: The principal survey was conducted from early February to early March 2008 by means of online questionnaires or by use of a paper. In total $N=511$ physicians' were asked by e-mail to participate in the survey; physicians without an e-mail address $(N=614)$ were sent a paper questionnaire. A total of 243 physicians answered the questionnaire; $76 \%$ male, $62 \%$ with a specialization in haematology.

Results: In the direct measurement of patients' preferences, physicians rated physical quality of life, rare side effects, and effectiveness aspects as most important attributes from the patients' perspective, followed by emotional quality of life and therapy-free-intervals. Especially further treatment options and dosage were more important to patients than physicians believed. In this case, the physicians had underestimated the importance of these attributes from the perspective of those affected. The experiment revealed that both groups agree that openness to further therapy options and maximum extension of life are the most important factors. Physicians ranked prolonged life expectancy as significantly more important than all other treatment attributes. A further treatment option was the second most important attribute and significant compared to the attributes breaks in therapy and physical quality of life.

Conclusions: Asking physicians about the patients' treatment preferences, the combination of direct assessment and Discrete-choice experiment proves to be a valid survey technique. Both the direct assessment of importance in order to rank the patients' perceptions and the Discrete-choice experiment provide important insights into the preference structure of patients with Multiple Myeloma. 


\section{Einleitung}

\subsection{Das Multiple Myelom}

Als Multiples Myelom wird das Auftreten einer gestörten Funktion von Plasmazellen an verschiedenen Stellen im Knochenmark bezeichnet. Das Multiple Myelom wird mehrheitlich einige Monate nach dem Auftreten der ersten Tumorzellen diagnostiziert, da die Symptome der Erkrankung sehr unspezifisch ausfallen [1-3]. Neben Müdigkeit und Schwäche werden vor allem die Knochenschmerzen im Brust- und Lendenwirbelbereich, von denen die meisten Betroffenen berichten, zunächst als rheumatisch, degenerativ oder osteoporotisch bedingt fehlinterpretiert und in der Folge fehlbehandelt [4-6].

In rund einem Fünftel aller Fälle wird das Multiple Myelom nur durch Zufall, d.h. im Rahmen einer anderen Untersuchung erkannt. Eine angemessene Behandlung setzt demzufolge vielfach erst dann ein, wenn die Krankheit bereits in ein fortgeschrittenes Stadium übergegangen ist. Aufgrund des bösartigen Charakters wird das Multiple Myelom der Gruppe der malignen Non-Hodgkin-Lymphome zugerechnet. Mit einer Inzidenz von etwa 4 auf 100.000 Einwohner pro Jahr in Europa und den USA gilt das Multiple Myelom als seltene Erkrankung. In Deutschland werden jährlichen etwa 3.500 Neuerkrankungen gezählt (Inzidenz), während derzeit insgesamt rund 12.000 Menschen von dieser Form von Krebs betroffen sind (Prävalenz). Das Multiple Myelom stellt eine Erkrankung des hohen Lebensalters dar. Die Erkrankung tritt in den meisten Fällen zwischen dem 60. und 80. Lebensjahr auf, wobei Männer häufiger betroffen sind als Frauen [7].

\section{2 Ärztliche Perspektive zu den Präferenzen}

Patientenzentrierte Versorgungsprogramme werden als ein wesentlicher Faktor in der Verbesserung der Qualität der Versorgung angesehen [8-10]. Qualitätsverbesserungen können insbesondere dann erzielt werden, wenn Ärzte die Präferenzen ihrer Patienten kennen und berücksichtigen.

Eine erste Studie zu den Patientenpräferenzen bei der Therapie des Multiplen Myelom im Sommer/Herbst 2007 untersuchte die Erwartungen und Vorstellungen von Patienten in Hinblick auf die Therapien bzw. Therapieeigenschaften des Multiplen Myeloms $(N=282)$ [11]. Im Mittelpunkt stand die Frage, welche Präferenzen Patienten auf der Basis ihrer Behandlungserfahrungen entwickeln und welche Ziele sie verfolgen. Eine Gegenüberstellung der Ärzteurteile und der Patientenpräferenzen findet sich im Aufsatz Mühlbacher, Nübling 2011 [12].

Im Gegensatz zu klinischen Studien, bei denen der primäre Fokus auf dem Nachweis der Wirksamkeit und Verträglichkeit von Medikamenten, Behandlungen oder Thera- pien liegt und Konstrukte der Lebensqualität nur einen geringen Korrekturfaktor bilden, sollen hier die Patientenpräferenzen für das Ganze von Patienten erfahrbare Spektrum von Therapieeigenschaften abgebildet werden.

Die Therapieeigenschaften umfassen dabei Faktoren, welche die körperliche, seelische und soziale Lebensqualität betreffen, wie die Nebenwirkungen der Behandlung, die Darreichungsform oder die Freiheitsgrade bei der Anwendung. Diese umfassende Bestimmung der Lebensqualität von Patienten ist deshalb von Interesse, weil sie eine Bewertung von Behandlungen auf einer breiteren Basis als auf der klassischen Grundlage medizinischer Indikatoren ermöglicht [13]. Insbesondere aber trägt sie dem Bestreben Rechnung, die Sichtweise der Betroffenen als Qualitätskriterium für die Bewertung von Therapieleistungen zu berücksichtigen (vgl. Patientenbeteiligung, Patient als Partner, Partizipative Entscheidungsfindung).

Präferenzen von Patienten werden bislang in der Regel bei den Entscheidungen über die Zulassung therapeutischer Maßnahmen nicht berücksichtigt. Damit bleibt weitgehend unbekannt, inwiefern die von der gesetzlichen Krankenversicherung bereitgestellten und durch die ärztlichen Entscheidungen veranlassten Behandlungen auch den Präferenzen der Versicherten entsprechen. Jedoch könnte sich profundes Wissen um den Patientennutzen insbesondere dann als ausschlaggebend für den Therapieerfolg erweisen, wenn Behandlungsmethoden nicht zweifelsfrei durch medizinische oder monetäre Kennzahlen zu unterscheiden sind [14]. Dieser Mangel an Information steht somit auch in deutlichem Widerspruch zu dem Ziel, effektive und effiziente Behandlungsformen zu garantieren. Präferenzstudien können diesem Informationsmangel entgegenwirken.

\subsection{Ziele der Studie}

Vor dem Hintergrund der patientenzentrierten Versorgung wurden die Patientenpräferenzen für die Therapie des Multiplen Myeloms ermittelt. Darüber hinaus soll hier die Sichtweise der Ärzte auf die Patientenpräferenzen dokumentiert werden. Die Untersuchung erfolgt mit Hilfe eines DiscreteChoice Experimentes (DCE). Ziel dieser Präferenz-Studie ist es zu ermitteln, welche Präferenzmuster Ärzte bei ihren Patienten vermuten, welche Merkmale sie als relevant für Patienten erachten und ob sich diese mit denen der Patienten selbst decken. Die Ergebnisse können dann genutzt werden, Differenzen in der Wahrnehmung zu adressieren und eine Orientierung bei der Versorgung von Myelom-Patienten an deren Präferenzen anzuregen.

\section{Studiendesign und Methode}

Die Studie gliederte sich in zwei Teile: ein Pretest als qualitative Vorstudie zur Absicherung der relevanten Merkmale 
und eine quantitative Hauptstudie zur Ermittlung der Patientenpräferenzen aus der Perspektive der ärztlichen Entscheider.

\subsection{Pretest als qualitative Vorstudie}

Eine umfangreiche qualitative Vorstudie bei Myelom-Patienten im Rahmen der ersten Präferenzstudie hatte die relevanten Aspekte der Therapie dargelegt. Die Indikatoren und Präferenzaspekte wurden durch Literaturrecherche und Fokusgruppen mit Patienten generiert und in einen quantitativen Fragebogen übersetzt. Für die Befragung der Ärzteschaft zu Patientenpräferenzen musste geklärt werden, ob Ärzte zusätzliche wichtige Faktoren bei ihren Patienten sehen oder vermuten, die in der Patientenstudie noch nicht genannt wurden. Dazu wurden die bereits für die Patienten ermittelten Eigenschaften innerhalb von Pretests mit Ärzten erneut validiert. Ziel des Pretests war daher die Überprüfung des mit Patienten erstellten Merkmalsraums für die Bewertung der Patientenpräferenzen bei Therapien des Multiplen Myeloms. Im November/Dezember 2007 wurde der Pretest bei den Ärzten durchgeführt. Während die Bereiche „Soziodemographie“ und „Rahmendaten“ des Fragebogens angepasst wurden, konnte der Fragebogenteil zu den Patientenpräferenzen identisch vorgelegt werden. Im Pretest wurden zusätzliche Textfelder eingefügt um den Ärzten Platz für Anmerkungen oder Vorschläge für aus ihrer Sicht zusätzlich relevante Aspekte zu geben. Insgesamt beteiligten sich 30 Probanden an dieser Voraberhebung per OnlineFragebogen. Die Auswertungen ergaben, dass die Fragen aus Sicht der Befragten eindeutig und verständlich waren, und sich keine wesentlichen Unterschiede ergaben.

\subsection{Soziodemographie und Rahmendaten}

Insgesamt nahmen im Zeitraum Februar bis März 2008213 Personen $(N=213)$ an der Haupterhebung teil und 30 weitere Teilnehmer während des Pretest. Die Gesamtfallzahl lag somit bei 243. Etwas über die Hälfte waren Papierfragebögen aus der Hauptstudie, knapp unter $50 \%$ waren direkte Eintragungen im Online-Fragebogen im Internet (davon $12 \%$ aus dem Pretest). Hinsichtlich des Geschlechtes der teilnehmenden Ärzteschaft ist in der realisierten Stichprobe ein deutlicher Überhang der Männer festzustellen, welche $75 \%$ der Befragten ausmachen. Die Verteilung der Stichprobe nach Dienstalter (gemessen am Jahr der Approbation) zeigt eine Häufung im Bereich von 1985-1996 (Mittelwert und Median $=1990, \mathrm{SD}=8,5$ Jahre). Das Durchschnittsdienstalter beträgt damit 17 Jahre, die Spanne reicht von einer Approbation vor 1970 (mehr als 37 Jahre) bis zum Jahr 2006 (1 Jahr Erfahrung). Rund drei von fünf befragten Ärztinnen und Ärzten $(62 \%)$ verfügen über den Facharzttitel Hämatologie.
Einschätzung des Kenntnisstand Patientenpräferenzen

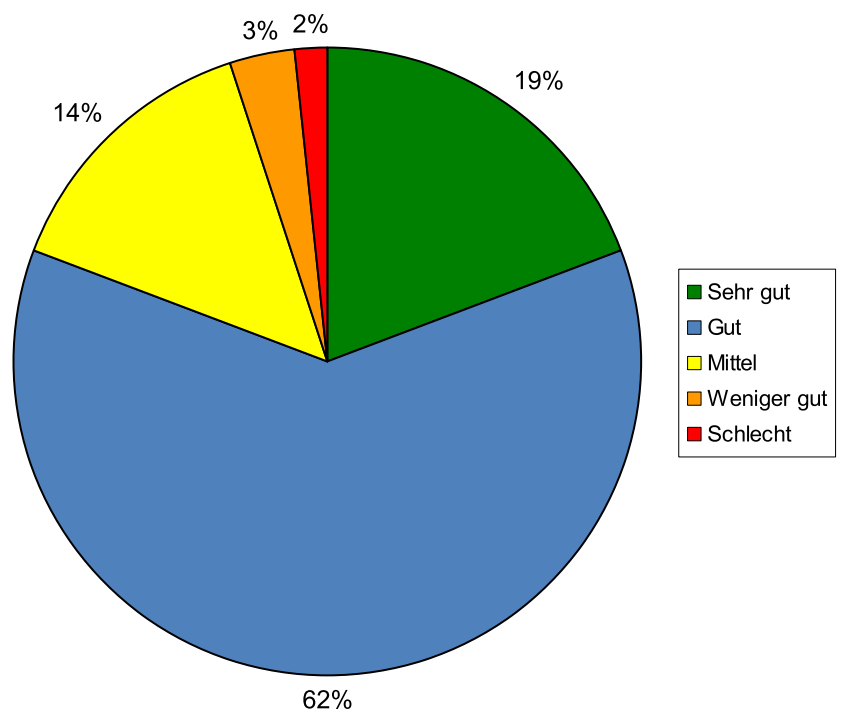

Abb. 1 Selbsteinschätzung der Kenntnis der Patientenpräferenzen

\subsection{Erfahrung und Selbsteinschätzung}

Ein kleiner Anteil von $4 \%$ der Befragten hat in den letzten 12 Monaten keine Patienten mit Multiplem Myelom behandelt oder beraten. Rund ein Viertel hat 1-10 Patienten behandelt, knapp $60 \%$ zwischen 11 und 50. $11 \%$ der Ärztinnen und Ärzte gaben an, in den letzten 12 Monaten mehr als 50 Myelom-Patienten behandelt zu haben.

Fast $20 \%$ der Ärzteschaft schätzten die eigenen Kenntnisse über die Patientenpräferenzen als sehr gut ein, weitere $62 \%$ als gut. $14 \%$ glauben, dass sie die Präferenzen mittelgut kennen, $5 \%$ sagen von sich, die Präferenzen der Patienten weniger gut oder gar nicht zu kennen (Abb. 1).

\subsection{Haupterhebung}

Der in der Haupterhebung verwendete Fragebogen gliederte sich in die folgenden Teilbereiche:

- Teil A: soziodemographische Parameter:

Geschlecht, Dienstalter, Facharzttitel Hämatologie

- Teil B:

Behandlungshäufigkeit, Selbsteinschätzung Kenntnis Patientenpräferenzen

- Teil C:

Wichtigkeitseinstufungen von Eigenschaften von Therapien des Multiplen Myeloms

o C1: direkte Abfrage von Wichtigkeiten bei insgesamt 17 Aspekten (5-stufige Likert-Skala)

o C2: Discrete-Choice Experiment (DCE) zur Messung der Patientenpräferenzen mit acht Paaren mit je acht Eigenschaften. 
Die Haupterhebung erfolgte von Anfang Februar bis Anfang März 2008 mittels Online-Fragebogen sowie als Paper-Pencil-Version, in beiden Fällen komplett anonym. Der Feldzugang bzw. die Rekrutierung der Probanden erfolgte über eine Ärztedatenbank der Janssen Cilag GmbH. Ärzte mit E-Mail-Adresse und E-Mail-Kontakterlaubnis $(N=511)$ wurden per E-Mail zur Teilnahme aufgefordert, Ärzte ohne E-Mail-Adresse $(N=614)$ bekamen einen Papier-Fragebogen samt portofreiem Rückumschlag zugesandt (der Online-Zugang war auf dem Fragebogen angegeben, so dass auch diese Gruppe den Online-Bogen nutzen konnte).

In der Auswertung kamen sowohl deskriptive Verfahren (Häufigkeitsauszählungen, statistische Kennwerte von Verteilungen) als auch bivariate Verfahren (Kreuztabellen, Mittelwertsvergleiche (ANOVA) und komplexe multivariate Verfahren (Faktorenanalyse (PCA), probit-Modelle, logitModelle) zur Anwendung.

Als Analysepakete wurden SPSS und STATA eingesetzt. Bei allen Analysen wurde $p<0,05$ (zweiseitig) als statistisch signifikant beurteilt.

\subsection{Discrete-Choice Experiment}

Patientenpräferenzen werden in der Gesundheitsökonomie zunehmend mit Hilfe der Discrete-Choice Analyse (DCE; oder auch Discrete-Choice Experiment) ermittelt [15]. Die DCE ist die wahlbasierte Form der Conjoint-Analyse, die durch die theoretischen Arbeiten von Lancaster (1966) [16] und McFadden (1974) [17] ermöglicht wurden. Bei der Discrete-Choice Analyse wird ein paarweiser Vergleich vorgenommen und eine (Aus-)Wahlentscheidung zwischen verschieden ausgestalteten Therapieangeboten von den Patienten (Probanden) gefordert [18].

Im zweiten Schritt wurden die Präferenzen in einem Discrete-Choice Experiment gemessen. Für das DiscreteChoice Experiment wurden den Befragten acht Auswahlentscheidungen zwischen jeweils zwei Therapien (A und B) in einem forced-Choice Design vorgelegt, die durch jeweils acht Qualitätsaspekte beschrieben wurden. Mittels spezieller Software (,SPEED“) wurde ein orthogonales Design mit 16 Auswahlsets erstellt. Die Bildung der acht Auswahlentscheidungen aus den 16 Auswahlsets erfolgte durch einen Zufallsgenerator (sequence generator). Es wurde Wert darauf gelegt, aus jedem inhaltlichen Bereich (Faktoren) mindestens einen Parameter zu berücksichtigen, um das ganze inhaltliche Spektrum der Präferenzen abzubilden (zu den verschiedenen Faktoren siehe Tab. 2). Die Auswahl und Formulierung der acht Eigenschaften für die Ärztestudie war identisch mit derjenigen in der Patientenstudie. Offensichtlich bedeutende Optionen, über die kein Dissenz unter den Patienten herrscht, wurden nicht berücksichtigt: (sofortige) Wirksamkeit, langanhaltende Effekte und Dosierungsflexibilität. Darüber hinaus wurde für die Befragung der Ärzte das Item „Behandlung durch den Arzt“ mit „Selbst-Applikation“ zusammengeführt. Die Parameter: Lebensdauer, Nebenwirkungen und Therapiepausen wurden im Experiment dreistufig vorgelegt, die Parameter körperliche, seelische und soziale Lebensqualität sowie Selbst-/Fremdbehandlung und Anschlusstherapien dichotom (Tab. 1). Aus diesen Merkmalen und Ausprägungen wurden 16 Alternativen generiert, die den Befragten in acht Paaren zur Wahl je von Alternative A oder B vorgelegt wurden.

\section{Ergebnisse}

\subsection{Therapieeigenschaften: Direkte Beurteilung und DCE}

Für die Patientenstudie waren auf der Basis bisheriger Forschung (Literaturstudie) und der eigenen qualitativen Vorstudie (Fokusgruppen) Merkmale gesammelt worden, die für die Bewertung von Therapien/Behandlungen aus Sicht der Patienten von Relevanz sind. Im Pretest der vorliegenden Ärztestudie waren diese Aspekte auf ihre Vollständigkeit hin überprüft worden. Auf Basis der Ergebnisse dieses Pretests wurde folgender, weiterer Aspekt in die direkte Abfrage der Wichtigkeiten aufgenommen, der von Ärzten als relevantes Kriterium aus Patientensicht angesehen wurde: „Wichtigkeit: Medikament beeinträchtigt Sexualität nicht".

Die Gesamtheit der Therapiemerkmale aus Patientensicht wurden den Ärzten zum einen in einer Direktabfrage (Rating), zum anderen in einem Discrete-ChoiceExperiment zur Bewertung vorgelegt. Die Direktabfrage hat gegenüber dem Discrete-Choice-Experiment den Vorteil, dass mehr Aspekte zur Bewertung vorgelegt werden können (in dieser Studie insgesamt 17). Ein Nachteil der Methode besteht darin, dass die Aspekte nicht simultan gegeneinander abgewogen werden müssen (Wahlentscheidungen), sodass Probanden alle Aspekte als „,besonders wichtig“ bewerten können (so genannte „Gratismeinungen“). Den Vorteil der direkten Gegenüberstellung bietet das DCE, indem Kombinationen mit Vor- und Nachteilen zur Bewertung vorgelegt werden und die Aspekte gegeneinander abgewogen werden müssen. Der methodische Nachteil ist, dass im DCE nur wenige Parameter bzw. wenige Stufen der Merkmale eingeschlossen werden können (hier: acht Merkmale mit 23 Stufen) $[11,19,20]$.

\subsection{Direkte Abfrage der Präferenzen}

Im ersten Teil der Befragung wurden den Ärzten insgesamt 17 Therapieeigenschaften zur Bewertung der Wichtigkeit auf einer 5-stufigen Likert-Skala von „,besonders wichtig“ bis ,nicht wichtig“ vorgelegt. Diese Angaben wurden 
Tab. 1 Merkmale und Ausprägungen im Discrete-Choice-Experiment

\begin{tabular}{|c|c|c|c|}
\hline Nr. & Merkmal & Anzahl Stufen & Ausprägungen \\
\hline 1 & Lebensdauer & 3 & $\begin{array}{l}\text { Durchschnittlich } \\
\text { Erhöht } \\
\text { Stark erhöht }\end{array}$ \\
\hline 2 & Nebenwirkungen & 3 & $\begin{array}{l}\text { Kurzfristig, vorübergehend } \\
\text { Mittelfristig } \\
\text { Langfristig, bleibend }\end{array}$ \\
\hline 3 & Behandlungsfreie Zeiten zwischen Therapielinien & 3 & $\begin{array}{l}\text { Keine } \\
\text { Kurzer Zeitraum } \\
\text { Längerer Zeitraum }\end{array}$ \\
\hline 4 & Körperliche Lage & 2 & $\begin{array}{l}\text { Eingeschränkte Beweglichkeit } \\
\text { Gute Beweglichkeit }\end{array}$ \\
\hline 5 & Seelische Lage & 2 & $\begin{array}{l}\text { Dauernd an Krankheit denken } \\
\text { Nicht dauernd an Krankheit denken }\end{array}$ \\
\hline 6 & Soziale Lage & 2 & $\begin{array}{l}\text { Keine langfristige Planung möglich } \\
\text { Langfristige Planung möglich }\end{array}$ \\
\hline 7 & Medikamentöse Behandlung & 2 & $\begin{array}{l}\text { Durch Arzt } \\
\text { Selbst }\end{array}$ \\
\hline 8 & Weitere Therapien & 2 & $\begin{array}{l}\text { Eventuell ausgeschlossen } \\
\text { Möglich }\end{array}$ \\
\hline
\end{tabular}

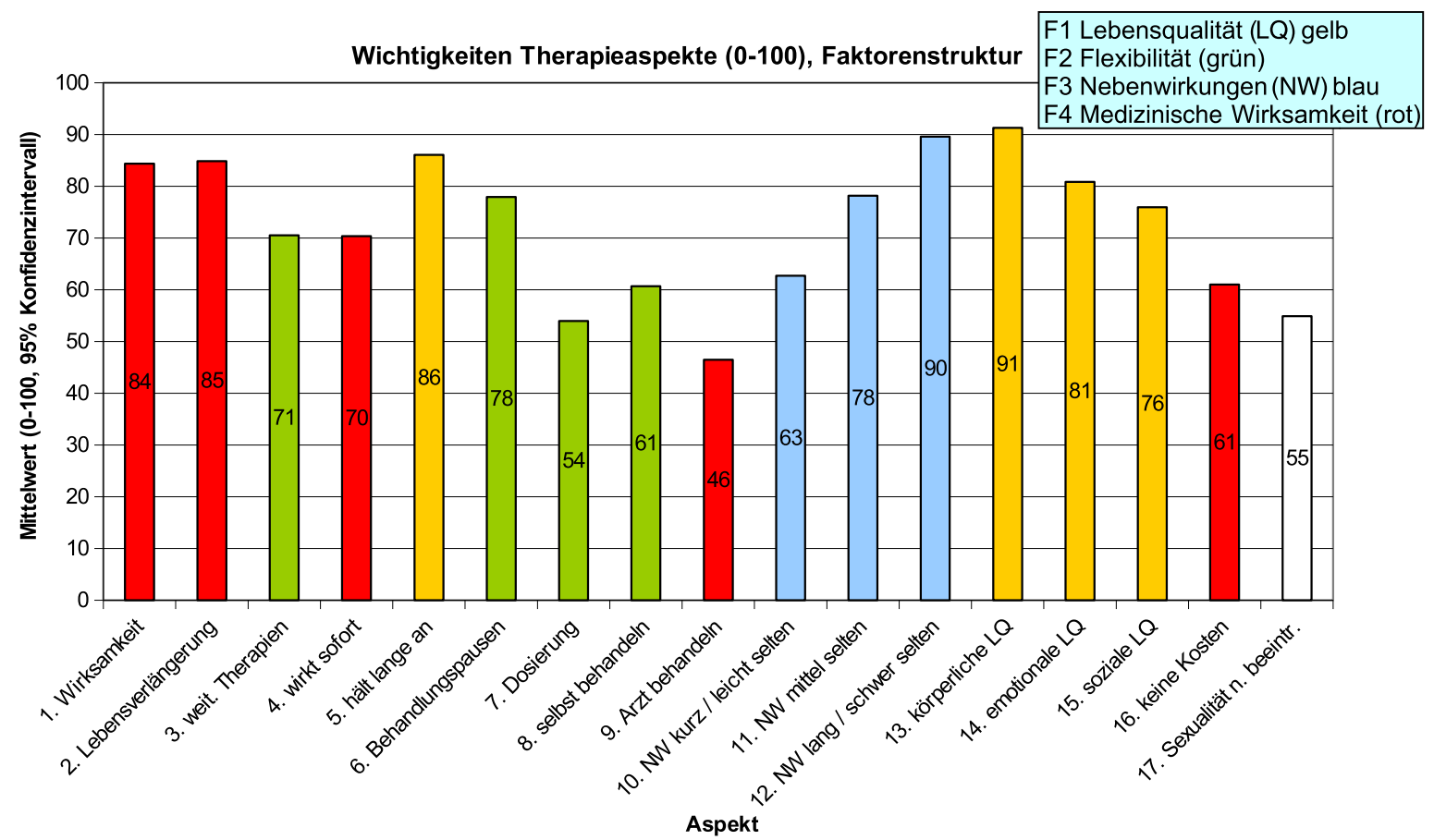

Abb. 2 Thematische Gruppierung der Wichtigkeiten der 17 Therapieeigenschaften in Faktorenstufen

auf einen Wertebereich von 0-100 transformiert (besonders wichtig $=100$, nicht wichtig $=0$ ). Abbildung 2 stellt die Mittelwerte der Wichtigkeitsbewertungen (und $95 \%$ Konfidenzintervall) auf der Basis aller Befragten dar. Hierbei wird deutlich, dass fast alle vorgelegten Aspekte relativ hohe Wichtigkeitswerte erzielen.
Die zwei von den Ärzten am höchsten bewerteten Aspekte (90 oder mehr Punkte) sind folgende:

- gute körperliche Lebensqualität (91)

- selten schwere/lang anhaltende Nebenwirkungen (90).

Am niedrigsten in der Relevanz wurden von den Ärzten die Aspekte 
Tab. 2 Faktorenstruktur und Faktorenladungen 16 Präferenzaspekte

\begin{tabular}{|c|c|c|c|c|}
\hline Item & $\mathrm{F} 1$ & $\mathrm{~F} 2$ & $\mathrm{~F} 3$ & F4 \\
\hline $\begin{array}{l}\text { Behandlung verbessert meine emotionale und seelische Verfassung (z. B. weniger } \\
\text { Ängste und Depressionen, weniger an Krankheit denken) (14) }\end{array}$ & $\mathbf{0 , 8 0 5}$ & & & \\
\hline $\begin{array}{l}\text { Behandlung verbessert meine sozialen Kontaktmöglichkeiten (z. B. langfristigere } \\
\text { Planung, Besuche etc.) (15) }\end{array}$ & $\mathbf{0 , 6 5 0}$ & 0,423 & & \\
\hline $\begin{array}{l}\text { Behandlung verbessert meine körperliche Verfassung (z. B. bessere Beweglichkeit, } \\
\text { Abnahme Schmerzen etc.) (13) }\end{array}$ & 0,645 & & & \\
\hline Wirkung der Behandlung hält lange an (5) & 0,490 & & & 0,337 \\
\hline Medikament kann je nach aktuellem Krankheitszustand dosiert werden (7) & & $\mathbf{0 , 8 0 8}$ & & \\
\hline $\begin{array}{l}\text { Medikamentöse Behandlung wird von mir selbst (zu Hause oder auch unterwegs) } \\
\text { durchgeführt (8) }\end{array}$ & & $\mathbf{0 , 5 8 3}$ & & \\
\hline Behandlung lässt weitere Therapiemöglichkeiten offen (für die Zukunft) (3) & & 0,565 & & \\
\hline Es gibt behandlungsfreie Zeiten/Behandlungspausen zwischen Therapielinien (6) & 0,304 & $\mathbf{0 , 4 2 1}$ & & \\
\hline $\begin{array}{l}\text { Mittelschwere oder mittellang anhaltende Nebenwirkungen (z. B. Fieber, Infekt, } \\
\text { reduzierte Empfindung für Lage, Bewegung, Vibration) durch Medikament sind } \\
\text { selten (11) }\end{array}$ & & & 0,904 & \\
\hline $\begin{array}{l}\text { Kurz anhaltende oder leichte Nebenwirkungen (z. B. Müdigkeit, Husten, zeitweises } \\
\text { Kribbeln) durch Medikament sind selten (10) }\end{array}$ & & 0,412 & 0,693 & \\
\hline $\begin{array}{l}\text { Langfristige und bleibende Nebenwirkungen (z. B. hohes Fieber, Verlust der } \\
\text { Empfindung für Lage, Bewegung, Vibration) durch Medikament sind selten (12) }\end{array}$ & 0,524 & & $\mathbf{0 , 5 3 1}$ & \\
\hline Behandlung gibt maximale Lebensverlängerung (2) & & & & $\mathbf{0 , 7 1 6}$ \\
\hline $\begin{array}{l}\text { Behandlung/Medikament hat sehr hohe Wirksamkeit (Remission, bessere } \\
\text { Laborwerte) (1) }\end{array}$ & & & & 0,633 \\
\hline Behandlung/Medikament wirkt sofort/spricht schnell an (4) & & & & $\mathbf{0 , 5 3 9}$ \\
\hline $\begin{array}{l}\text { Behandlung/Medikament verursacht mir keine zusätzlichen Kosten (keine } \\
\text { Zuzahlung) (16) }\end{array}$ & & & & $\mathbf{0 , 4 2 9}$ \\
\hline $\begin{array}{l}\text { Medikamentöse Behandlung wird durch Arzt in Praxis oder Krankenhaus } \\
\text { durchgeführt (9) }\end{array}$ & & 0,327 & & $\mathbf{0 , 3 5 6}$ \\
\hline
\end{tabular}

- Behandlung durch Arzt oder Krankenhaus (46)

- flexible Dosierung möglich (54) sowie

- Sexualität nicht beeinträchtigt (55)

bewertet.

\subsection{Gruppierung Präferenzen}

Eine Annahme dieser Studie war, dass die abgefragten Präferenzen nicht völlig unabhängig voneinander sind, sondern sich zu größeren Inhaltsbereichen mit einander ähnlichen Aspekten zusammenfassen lassen. Diese Vermutung wurde mittels einer Faktorenanalyse (Hauptkomponentenanalyse (PCA), Faktorenzahl nach Kaiser-Kriterium und VarimaxRotation) überprüft, wobei nur die 16 Aspekte einbezogen wurden, die auch in der Patientenbefragung enthalten waren.

In der explorativen und konfirmatorischen Faktorenanalyse ergab sich eine 4-Faktorenlösung mit 50,2 \% erklärter Varianz. Bis auf zwei Ausnahmen (Aspekte 9 und 16) wurden Kommunalitäten über 0,30 erreicht.

Je vier Aspekte luden primär auf den Faktoren 1 und 2, drei Aspekte sind primär Faktor 3 zugeordnet und fünf
Aspekte dem Faktor 4 (Tab. 1, Primär-Ladungen fett gedruckt). Die Kommunalität gibt an, wie gut jeder Einzelaspekt durch die Faktoren insgesamt abgebildet wird, während die Primärladung eines Aspekts die jeweils höchste Ladung beschreibt und somit die primäre Zuordnung eines Aspekts zu einem Faktor vorgibt (zur Methodik siehe z. B. Backhaus et al. [19] und Bortz [20]).

Die rotierte Faktorenmatrix samt aller Ladungen $>0,30$ sowie die Zuordnung der Items zu den vier Faktoren sind in Tab. 2 wiedergegeben.

Die Nummerierung der Faktoren trifft keine Aussage über deren Relevanz für die Befragten, sondern ist primär von der Anzahl der Items und der Höhe der Itemladungen auf dem Faktor abhängig.

Die Faktoren oder übergeordneten Präferenzdimensionen wurden semantisch mit folgenden Titeln beschrieben:

- Faktor 1: Lebensqualität (körperlich, seelisch, sozial)

- Faktor 2: Flexibilität (inkl. Anschlussfähigkeit, Behandlungspausen)

- Faktor 3: Nebenwirkungen

- Faktor 4: medizinische Wirksamkeit 
Ähnliche Gruppierungen hatten sich auch in der Patientenstudie ergeben [11].

\subsection{Gruppenunterschiede in Präferenzen}

Im Rahmen der Studie wurden die Ergebnisse der direkten Befragung hinsichtlich Heterogenitäten untersucht. In entsprechenden Varianzanalysen (ANOVA) wurde geprüft, ob die sechs Parameter,

- Pretest-Hauptstudie,

- Geschlecht Arzt/Ärztin,

- Dienstalter Arzt/Ärztin,

- Facharzttitel Hämatologie ja-nein,

- Anzahl Multiples Myelom-Patienten in letzten 12 Monaten und

- Selbsteinschätzung der Kenntnis der Patientenpräferenzen

einen Einfluss auf die Einschätzung der Patientenpräferenzen ausüben.

Der Parameter Pretest-Hauptstudie zeigte bei keinem der 16 untersuchten Aspekte signifikante Unterschiede (Aspekt 17 wurde erst in der Hauptstudie neu integriert), was die $\mathrm{Zu}$ lässigkeit der gemeinsamen Analyse der Daten aus Pretest und Hauptstudie untermauerte.

Das Geschlecht der Befragten zeigte bei einem der 17 Aspekte der Hauptstudie einen signifikanten Unterschied: Männer bewerten die Wichtigkeit der Verbesserung der emotionalen Lage höher als Frauen (82 gegenüber 76 Punkten).

Bezüglich des Dienstalters wurden durch Teilung am Median zwei etwa gleich große Gruppen gebildet: Approbation vor 1989 (18 Jahre und mehr) und Approbation 1990 und später (max. 17 Jahre). Bei zwei Aspekten zeigen sich signifikante Unterschiede: Die Ärztinnen und Ärzte mit dem höheren Dienstalter geben der flexiblen Dosierung (Item 7) und der Behandlung durch den Arzt (Item 9) jeweils ein höheres Gewicht (Item 7: 59 zu 50 Punkte; Item 9: 51 zu 42 Punkte).

Der Parameter Facharzttitel Hämatologie steht in einem Zusammenhang mit der Beantwortung dreier Items. Inhaber des Facharzttitels geben der maximalen Lebensverlängerung ( 87 zu 81 Punkte) ein höheres Gewicht im Vergleich zu ärztlichen Kollegen, die keinen Facharzttitel haben. Dagegen schätzen Fachärztinnen und Fachärzte die Relevanz der Behandlung durch den Arzt (44 zu 50 Punkte) und des nicht beeinträchtigten Sexuallebens (52 zu 60 Punkte) als weniger hoch ein.

Die Anzahl der behandelten Patienten mit Multiplem Myelom steht signifikant im Zusammenhang mit der Einschätzung von vier Aspekten: Vielbehandler mit mehr als 50 Patienten schätzen die maximale Lebensverlängerung höher ein (92 zu 85 Punkten im Gesamtdurchschnitt). Dagegen stufen die weniger Erfahrenen (bis maximal 10 Patienten) die Relevanz der flexiblen Dosierung höher ein (> 60 gegenüber ca. 50 Punkten bei den Vielbehandlern).

Schwächer zeigen sich die Zusammenhänge bei mittelschweren Nebenwirkungen, die von denjenigen ohne Patienten in den letzten 12 Monaten als wichtiger eingeschätzt werden als von Kollegen mit betroffenen Patienten. Bei der Frage nach der Vermeidung von Zuzahlungen zeigt sich, dass dieses Item, in den mittleren Gruppen mit 1-10 bzw. 11-50 Patienten höher bewertet wird als in den Gruppen ohne bzw. mit mehr als 50 Patienten. Befragte, die sich selbst als gute Kenner der Patientenpräferenzen einschätzen, geben den Items ,maximale Lebensverlängerung“, „,behandlungsfreie Zeiten“, „flexible Dosierung“ und „,keine Zuzahlung“ jeweils signifikant mehr Gewicht.

\subsection{Präferenzen im Discrete-Choice Experiment}

Insgesamt lagen von 243 Personen $(N=243) 1931$ gültige Beobachtungen $(N=1931)$ vor. Bei vollständiger Bewertung aller Paare ergeben sich pro Person acht Beobachtungen, einige wenige Probanden haben aber gar keine oder nicht alle Paare bewertet. Im Durchschnitt wurden von den 243 Probanden, die mindestens einen Paarvergleich absolvierten, 7,98 Paare bewertet (Spannweite 6-8). Als Schlussmodell wurde ein Random Effects Probit Model erstellt, das die Teilabhängigkeit von Beobachtungen derselben Person bei den Parameterschätzungen mit berücksichtigt. Dieses Vorgehen wurde durch ein hoch signifikanten Chibar ${ }^{2}$ unterstützt.

Für die Analyse wurden die gewählten Merkmale und ihre Stufen Effekt-codiert: Grundsätzlich wurde die inhaltlich als „,besser“" klassifizierte Eigenschaft positiv kodiert, z. B. bei körperlicher Lebensqualität die Ausprägung ,nicht eingeschränkt“", während ,eingeschränkt" den negativen Pol markiert. Da es bei dem Item „Medikamentöse Behandlung selbst oder durch Arzt" keine a priori Hypothese über die Bewertung der Pole gab, wurde hier ,selbst“ als positiv und „Arzt“" als negativ kodiert.

Für einige Merkmalausprägungen konnten keine gültigen Schätzungen erstellt werden, da Kollinearität vorlag. Dies betraf - wie bei den Patienten auch - die mittlere Kategorie von Lebensdauer, die höchste Kategorie des Parameters Nebenwirkungen, die mittlere Kategorie des Parameters Behandlungspausen sowie die soziale Lebensqualität.

Wie die Ergebnisse in Tab. 3 zeigen, wird die Wahl einer Therapie bzw. eines Medikaments aus Patientensicht nach Meinung der Ärzte von verschiedenen Eigenschaften unterschiedlich stark beeinflusst. Den numerisch stärksten Einfluss übt aus Sicht der Ärzteschaft die maximale Erhöhung der Lebensdauer aus. Dahinter folgen als Entscheidungsfaktoren die Aspekte der Möglichkeit weiterer Anschlusstherapien und der Selbstbehandlung sowie die Merkmale seelische Lebensqualität (nicht immer an Krankheit denken) 
Tab. 3 Ergebnisse Random Effects Model (Discrete Choice Experiment)

\begin{tabular}{|c|c|c|c|c|c|c|}
\hline Item & Merkmal + Ausprägung & Koeffizient & SE (Koeff.) & $95 \%$ KI (Koeff.) & Sig. & Odds Ratio \\
\hline \multirow[t]{3}{*}{1} & Lebensdauer durchschnittlich & $-0,778$ & 0,052 & & $* * *$ & 2,177 \\
\hline & Lebensdauer erhöht & - & - & & - & \\
\hline & Lebensdauer stark erhöht & 0,778 & 0,052 & $0,68-0,88$ & $* * *$ & \\
\hline \multirow[t]{3}{*}{2} & Nebenwirkungen leicht/kurz & - & - & & - & ns \\
\hline & Nebenwirkungen mittel & $-0,049$ & 0,049 & & ns & \\
\hline & Nebenwirkungen schwer/lang & 0,049 & 0,049 & $-0,14-0,05$ & ns & \\
\hline \multirow[t]{3}{*}{3} & Keine Behandlungspausen & $-0,193$ & 0,040 & & $* * *$ & 1,213 \\
\hline & Kurze Behandlungspausen & - & - & & - & \\
\hline & Längere Behandlungspausen & 0,193 & 0,040 & $0,11-0,27$ & $* * *$ & \\
\hline \multirow[t]{2}{*}{4} & Körperliche Lage eingeschränkt & $-0,098$ & 0,037 & & $* *$ & 1,103 \\
\hline & Körperliche Lage nicht eingeschränkt & 0,098 & 0,037 & $0,03-0,17$ & $* *$ & \\
\hline \multirow[t]{2}{*}{5} & Immer an Krankheit denken & $-0,218$ & 0,067 & & $* * *$ & 1,244 \\
\hline & Nicht immer an Krankheit denken & 0,218 & 0,067 & $0,09-0,35$ & $* * *$ & \\
\hline 6 & Soziale Lage & - & - & - & - & - \\
\hline \multirow[t]{2}{*}{7} & Medikamentöse Behandlung Arzt & $-0,272$ & 0,078 & & $* * *$ & 1,313 \\
\hline & Medikamentöse Behandlung selbst & 0,272 & 0,078 & $0,12-0,43$ & $* * *$ & \\
\hline \multirow[t]{3}{*}{8} & Weitere Therapien evtl. ausgeschlossen & $-0,423$ & 0,039 & & $* * *$ & 1,527 \\
\hline & Weitere Therapien möglich & 0,423 & 0,039 & $0,35-0,50$ & $* * *$ & \\
\hline & Modell-Konstante & $-0,175$ & 0,066 & $-0,30-0,05$ & $* *$ & \\
\hline
\end{tabular}

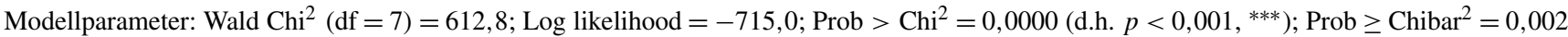
(d.h. $p<0,01,{ }^{* *}$ )

${ }^{* * *} p<0,001,{ }^{* *} p<0,01,{ }^{*} p<0,05$

und Behandlungspausen. Aus ärztlicher Einschätzung der Wahl einer Therapie oder eines Medikaments durch die Patienten wird der Aspekt körperliche Lebensqualität als weniger bedeutsam eingeschätzt, ist jedoch noch statistisch signifikant. Keine zusätzliche signifikante Rolle nimmt im Modell dagegen die Ausprägung der Nebenwirkungen ein. Zur Relevanz der sozialen Lebensqualität kann aufgrund der Kollinearitäten keine Aussage gemacht werden.

Der Unterschied zwischen den Wichtigkeiten kann spätestens dann als signifikant angesehen werden, wenn sich ihre Konfidenzintervalle (95\% KI) nicht mehr berühren bzw. überlappen. Demnach ist die Wichtigkeit des Items „Lebensdauer“ signifikant höher als die jeder der anderen Eigenschaften. Es folgt Item ,weitere Therapiemöglichkeiten“, das signifikant noch wichtiger als die Items „Behandlungsfreie Zeiten zwischen den Therapielinien“ und „Körperliche Lage“ ist. Zwischen „(medikamentöser) Selbstbehandlung“ und „nicht an die Krankheit denken (seelische Lage)“, ist kein signifikanter Unterschied feststellbar, da sich die Konfidenzintervalle deutlich überschneiden (Abb. 3).

Der Vergleich der Aspekte „Weitere Therapien“ und „seelische Lage (nicht an die Krankheit denken)“ führt zu einer Berührung beider Konfidenzintervalle im Wert 0,35. Dieses Ergebnis ist somit auf Basis des hier spezifizierten
Modells und des ex-ante festgelegten Chow-Tests nicht signifikant. Eine höhere Fallzahl hätte zu einem eindeutigeren Ergebnis führen können. Eine ex-post geänderte Auswahl des Testverfahren zwecks Erzielung eines signifikanten Ergebnisses wird nicht empfohlen. Die verschiedenen Aspekte können auch in Form der odds ratios dargestellt werden.

Bei der Darstellung der Entscheidungswahrscheinlichkeiten entfallen auf das Item maximale Lebensdauer 2,177 der Erklärkraft innerhalb der Entscheidung gefolgt von „,weitere Therapiemöglichkeiten“ mit 1,527. Die Erklärkraft in Bezug auf Odds Ratios bezieht sich hierbei auf die Annahme, dass die berechneten Odds einen bestimmten Anteil der Entscheidung verdeutlichen. Je größer der Wert, desto größer ist das Gewicht des jeweiligen Attributes innerhalb der Entscheidung. Vereinfacht: Je größer der Wert, desto größer die Wahrscheinlichkeit, dass die Alternative gewählt wird, wenn dieses Attribut in der Auswahlalternative enthalten ist. Das Item „Soziale Lage“ konnte nicht geschätzt werden, Item „Nebenwirkungen“ ist nicht signifikant.

Eine geringere Erklärkraft auf die Entscheidung weisen die Aspekte auf, die Freiräume im weitesten Sinne beschreiben: Medikamentenanwendung auch in Eigenregie möglich sowie seelische Lebensqualität: nicht dauernd an Krankheit denken. Auf das Item lange Behandlungspausen zwischen den Therapien entfallen 1,231, während das Item körper- 
Abb. 3 Odds Ratios der Entscheidungsparameter

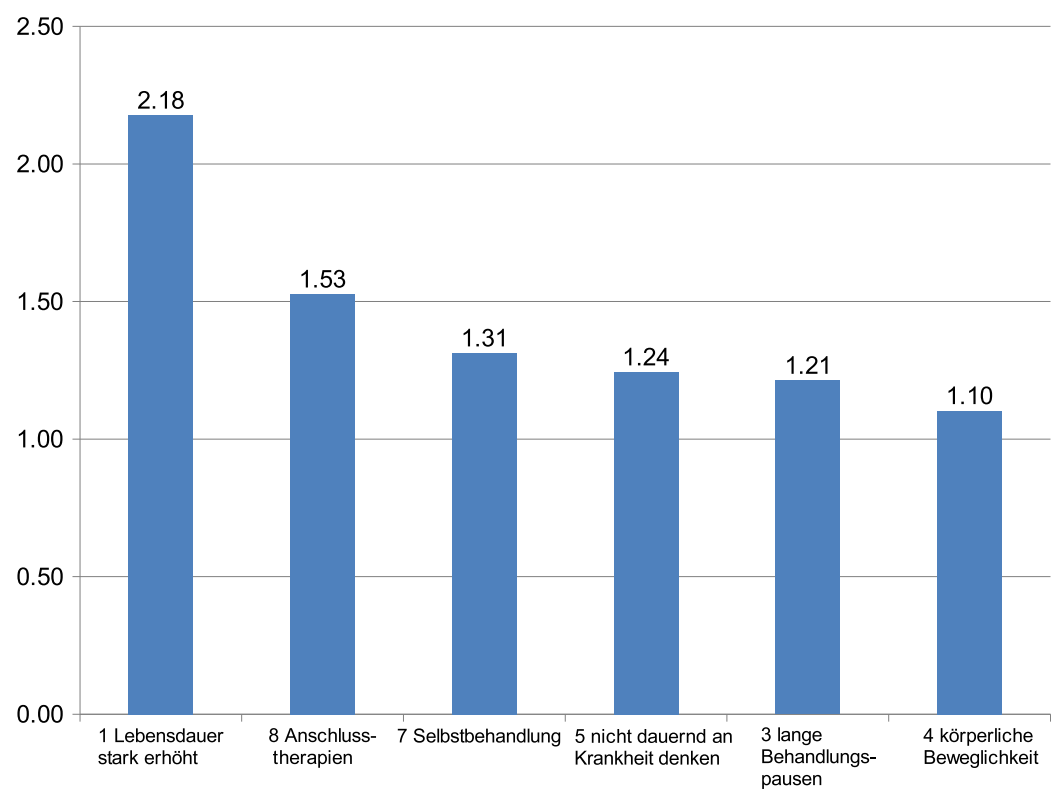

liche Beweglichkeit noch eine Erklärkraft auf die Entscheidung von 1,103 zeigt.

In zusätzlichen Analysen wurde geprüft, ob die Parameter aus Teil A und B (Geschlecht, Dienstalter, Facharzttitel Hämatologie, Anzahl der Patienten mit Multiplem Myelom und Selbsteinschätzung der Kenntnis der Patientenpräferenzen) diese Präferenzstruktur wesentlich modifizieren. Es stellte sich die Frage, ob z. B. die bei Patienten vermuteten Therapie-Präferenzen bei Ärztinnen wesentlich anders gewichtet sind als bei Ärzten. Diese Rahmendaten waren weder als unabhängige Einzelvariablen noch als Interaktionen mit den Therapie-Eigenschaften signifikante Prädiktoren für die Wahlentscheidungen. Dies gilt auch, wenn die acht Wahlmöglichkeiten separat betrachtet werden.

Im Anschluss an die acht Paarvergleiche des DCE (am Ende des Fragebogens) wurden die Befragten um eine Bewertung der Schwierigkeit bei der Durchführung der Paarvergleiche des DCE gebeten. Nur $2 \%$ fanden es sehr schwierig, weitere $17 \%$ eher schwierig, $46 \%$ kreuzten die Mitte an (teils-teils), $27 \%$ fanden es eher nicht schwierig und $8 \%$ hatten nach eigenem Bekunden gar keine Probleme mit dem Ausfüllen des DCE. Im Gesamtbild gilt das DCE bei der Ärzteschaft also als praktikabel.

\section{Diskussion}

Bei der Bewertung medizinischer Dienstleitungen (z. B. Medikamente oder Therapien) wird die Sicht der Betroffenen oft nur ungenügend berücksichtigt.

Gerade vor dem Hintergrund von Stichworten wie „Gesundheitskompetenz“, „Patientenpartizipation“ und „Shared decision making" sollte die Analyse und Berücksichtigung der Präferenzen und Wünsche der Patienten bezüglich der Therapien und Medikamente selbstverständlich sein. Aus diesem Grund wurden in einer ersten Studie (2007, $N=$ 282) die Patientenpräferenzen bei der Behandlung des Multiplen Myeloms durch eine Befragung betroffener Patienten und Patientinnen analysiert [11]. Zum Einsatz kamen die direkte Abfrage von Präferenzen sowie ein Discrete-Choice Experiment. Wichtig ist ergänzend zu ermitteln, ob und wie gut die Leistungserbringer die Präferenzen ihrer Patienten kennen. Die behandelnden Ärzte wurden daher mit demselben Instrument befragt, wobei ihre Einschätzung der Präferenzen der Patienten im Mittelpunkt stand.

\subsection{Direkte Messung Präferenzen}

Die Ärzte, wie auch die Patienten, messen medizinischen Parametern wie maximaler Lebensverlängerung, hoher Wirksamkeit und langer Wirkungsdauer die höchste Priorität bei. Dieser Fakt ist nicht überraschend, handelt es sich hierbei doch um „Muss-Faktoren“ für eine Behandlung oder ein Medikament (Abb. 4).

Aus Patientensicht besitzt der Aspekt, dass durch die aktuelle Therapie weitere, zukünftige Therapiemöglichkeiten nicht ausgeschlossen werden dürfen, ebenfalls große Bedeutung. Dies scheint ein bei der Therapie des Multiplen Myeloms spezifischer Aspekt zu sein, der von den Ärzten nicht an so exponierter Stelle gesehen wird. Etwas nachgelagert aus Patientensicht, aber trotzdem mit hohen Werten in der Wichtigkeit, folgen Merkmale der Sicherstellung der körperlichen Lebensqualität und der Vermeidung langfristiger (irreversibler) Nebenwirkungen. Diese beiden Aspekte rangieren bei der Einschätzung der Relevanz durch die Ärzte dagegen auf den vordersten Plätzen. 
Abb. 4 Direkte Abfrage: Wichtigkeit Therapieaspekte aus Sicht Patienten und Ärzte

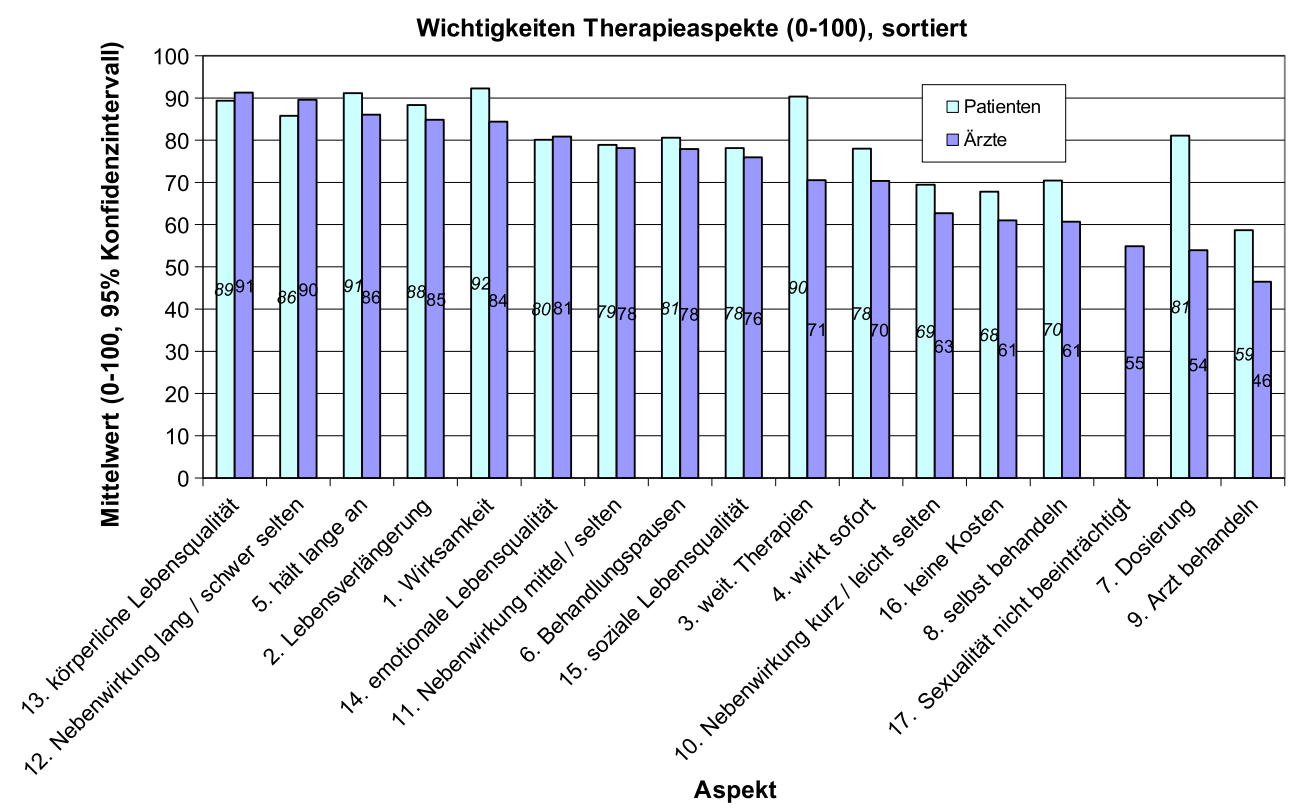

Auch die emotionale Lebensqualität und die Möglichkeit von Behandlungspausen haben einen hohen Stellenwert für die Patienten - sowohl in deren eigener Einschätzung als auch in der Einschätzung der Ärzte.

Weniger wichtig scheinen die soziale Lebensqualität, die Frage durch wen die Medikamentenvergabe/Behandlung erfolgt (selbst oder Arzt) und Kostenfragen zu sein, wobei sich beide Gruppen in ihrer Bewertung weitgehend einig sind. Auffällige Diskrepanz herrscht jedoch bei der Bewertung des Flexibilitätsaspektes Dosierung: diesen finden Patienten laut Eigenangabe weitaus wichtiger als Ärzte das in der Fremdeinschätzung glauben.

Methodisch muss bei den Heterogenitätstests beachtet werden, dass die Vielzahl der Tests das Artefaktrisiko erhöht. Zur Auswertung der Daten wurden 101 statistische Signifikanztests $(17 \times 5+16 \times 1=101)$ durchgeführt, sodass das Artefaktrisiko wegen multiplen Testens ansteigt. Während bei einem Signifikanzniveau von $p<0,05$ im Mittel fünf zufällig signifikante Ergebnisse bei 100 Tests erwartet werden, wurden in diesem Fall 14 signifikante Differenzen deutlich. Dies lässt vermuten, dass einige Differenzen reale Unterschiede abbilden. Auffällig ist, dass die erfahreneren Befragten (Facharzttitel, viele Patienten, hohe Selbsteinschätzung eigener Kenntnisse) die maximale Lebensverlängerung höher gewichten als ihre Kollegen. Insgesamt sind die Unterschiede in den Präferenzen aber eher gering zu bewerten. Die durch die geprüften Rahmendaten erklärte Varianz $\left(\right.$ eta $\left.^{2}\right)$ erreicht in keinem Fall mehr als $7 \%$.

\subsection{Messung Präferenzen im DCE}

Mit einem Discrete-Choice-Modell konnten die Teilnutzen der einzelnen Aspekte für die Wahlentscheidung ermittelt werden (Abb. 5).
Die höchste Relevanz für die Wahl der Patienten selbst hatte der Aspekt, ob weitere Behandlungen möglich blieben, gefolgt von der Sicherstellung einer möglichst stark erhöhten Lebensdauer. Lagen diese Therapieeigenschaften vor, wurden die entsprechenden Alternativen deutlich präferiert (zusammen $61 \%$ der Entscheidungsrelevanz).

Auch in der Fremdeinschätzung der Ärzte lagen diese beiden Parameter deutlich vorn, allerdings in umgekehrter Reihenfolge. In der Sicht der Ärzte ist der primäre Entscheidungsparameter der Patienten die maximal verlängerte Lebensdauer, mit Abstand gefolgt von der Möglichkeit von Anschlusstherapien. Zusammen machen diese beiden Parameter $60 \%$ Entscheidungsrelevanz aus.

Weiterhin entscheidungsrelevant sind aus Ärztesicht die Aspekte der Selbstbehandlung und einer guten seelischen Lebensqualität (nicht immer an Krankheit denken). Die Patienten gewichten die seelische Lebensqualität höher (Unterschied Ärzte-Patienten knapp signifikant, $p=0,03$ ), während nach Auffassung der Ärzte, der Möglichkeit der Selbstbehandlung ein höheres Gewicht eingeräumt wird (Unterschied Patienten-Ärzte nicht signifikant).

Bei der Bewertung der Möglichkeit längerer Behandlungspausen zwischen den Therapielinien sind sich beide Gruppen einig und räumen dem Aspekt gleiche Entscheidungsrelevanz ein. Ebenso wird die Relevanz der körperlichen Lebensqualität von beiden Gruppen ähnlich untergeordnet gesehen. Der Parameter Nebenwirkungen ist im DCE für beide Gruppen nicht signifikant. Für den Parameter soziale Lebensqualität konnte in beiden Modellen kein Schätzwert ermittelt werden.

Für beide befragten Gruppen ergaben sich im DCE generalisierbare Präferenzmodelle, die nicht wesentlich durch 
Abb. 5 Vergleich Ärzte und Patienten, 6 schätzbare signifikante Attribute, jeweils positiver Pol, random effects model

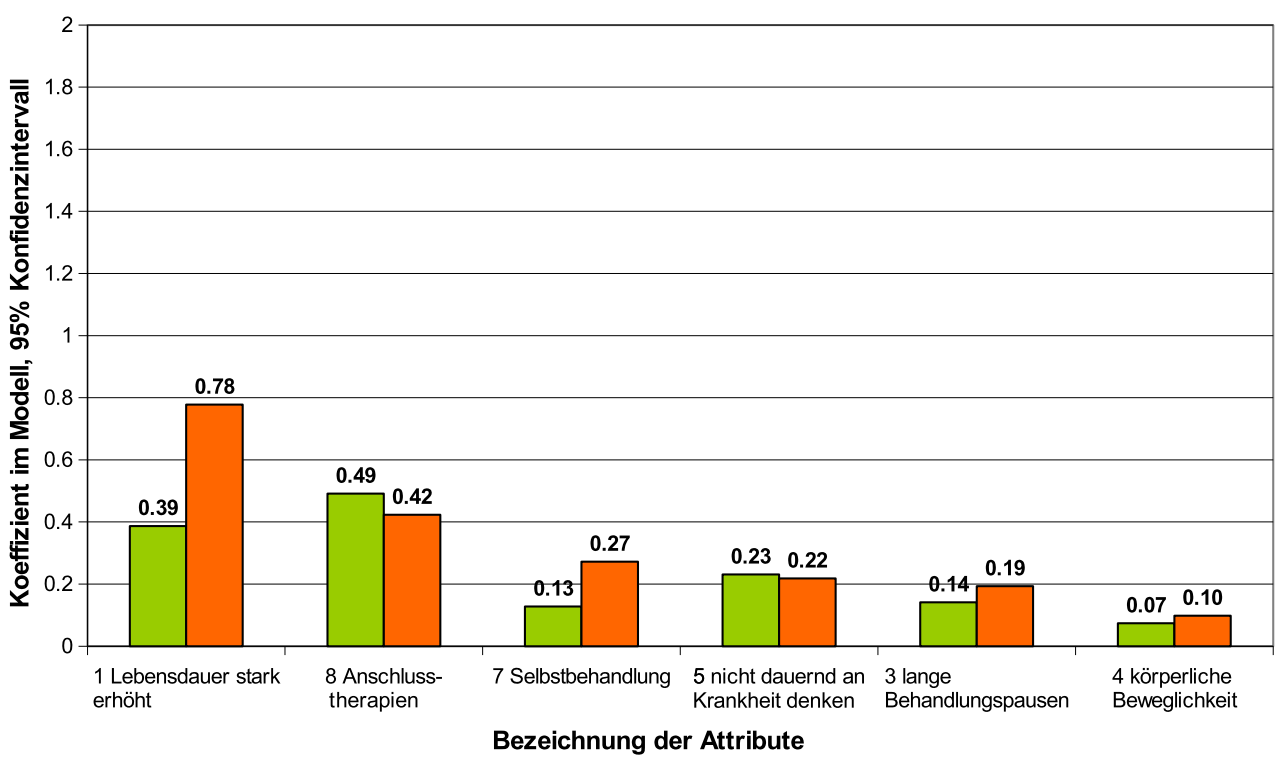

soziodemographische Parameter (Alter, Geschlecht etc.) beeinflusst wurden.

Allerdings erscheint es sinnvoll, die Präferenzstruktur für Ärzteschaft und für Patienten getrennt zu berechnen und darzustellen. Wie der entsprechende Chow-Test für alle acht Modellkoeffizienten zeigt $\left(\mathrm{Chi}^{2}=80,5 ; \mathrm{df}=3 ; p<\right.$ 0,001 ), ist die Abfolge und Gewichtung der einzelnen Therapieeigenschaften in ihrer Gesamtheit bei Ärzten und Patienten trotz einiger Ähnlichkeiten signifikant unterschiedlich.

Insgesamt, direkte Wichtigkeitsmessung und DCE zusammen betrachtet, besteht bei den Einschätzung der Patientenprioritäten für die meisten Aspekte weitgehende Einigkeit: was Patienten besonders wichtig finden, von dem glauben auch die Ärzte, dass das für ihre Patienten besonders relevant ist. Entgegengesetzt: Was Patienten als weniger wichtig klassifizieren, wird auch in der Ärzteeinschätzung der Patientenwünsche als untergeordnet bewertet. Zudem sind sich sowohl die Patienten untereinander als auch die Ärzteschaft untereinander weitgehend einig.

\subsection{Methodenvergleich direkte Messung - DCE}

In beiden Studiengruppen decken sich die Ergebnisse der beiden methodischen Herangehensweisen weitgehend. Markante Ausnahmen sind die geringere Gewichtung der körperlichen Lebensqualität und der Nebenwirkungen im DCE im Vergleich zur direkten Wichtigkeitsbewertung. Die geringere Einstufung von Nebenwirkungen als entscheidungsrelevanter Faktor im DCE kann auch dadurch begründet sein, dass die Aspekte in engem Zusammenhang stehen und sich gegenseitig teilweise bedingen.

\section{Schlussfolgerung}

Um eine patientenzentrierte Versorgung aufzubauen oder zu garantieren, ist die Ermittlung der Patientenpräferenzen unerlässlich. Hier ist zunächst die Meinung der Patienten selbst relevant. Daneben ist es jedoch auch wichtig zu analysieren, ob die Ärzte als primäre Versorger diese Präferenzen ähnlich einschätzen bzw. kennen oder ob sie aus ihrer Erfahrung andere Präferenzmuster bei ihren Patienten vermuten. Die Information über die Patientenpräferenzen wird als weitere Informationsquelle und Form der Evidenz angesehen, die von Ärzten und anderen Stakeholdern in Entscheidungsprozessen berücksichtigt werden sollten. Die hier aufgezeigten Studienergebnisse könnten zur Verbesserung der patientenzentrierten Versorgung beitragen, so dass diese in politischen und medizinischen Entscheidungen berücksichtigt werden.

Unsere Studien haben gezeigt, dass die Kombination von direkter Erhebung von Wichtigkeiten und der Erhebung in wahlbasierten Verfahren (DCE) sinnvoll und fruchtbar ist. Die Verfahren führen zu vergleichbaren Resultaten, wobei die direkte Messung mehr zusätzliche Aspekte berücksichtigt und das DCE die wichtigsten Aspekte besser gegeneinander abwägen lassen kann.

Zukünftige Studien sollten klären, ob diese methodischen Befunde (Selbsteinschätzung versus Fremdeinschätzung, direkte Messung versus DCE Messung) auch bei anderen Settings, Indikationen und Fragestellungen Gültigkeit haben.

Insgesamt ergaben sich nur geringe Unterschiede in den Einschätzungen, so dass das vorgestellte Präferenzmodell als allgemein gültig für Ärztinnen und Ärzte bei der Einschätzung der Präferenzen von Patienten mit Multiplem Myelom gelten kann. 
Danksagung Unser besonderer Dank gilt dem Selbsthilfenetzwerk APMM sowie der Deutsche Leukämie- und Lymphom-Hilfe für ihre Unterstützung in der Generierung der qualitativen und quantitativen Daten.

Für diese Studie wurden von der Ortho Biotech, Division of Janssen Cilag GmbH Neuss Forschungsgelder zur Verfügung gestellt.

Autorenerklärung/Interessenkonflikte: Die Autoren haben das Design der Studie entwickelt und die Datengenerierung, statistische Auswertung sowie die Interpretation der Daten selbstständig durchführt. Beide Autoren waren an der Interpretation der Daten und der kritischen Revision des Manuskripts beteiligt, haben das fertige Manuskript gelesen und genehmigt.

Open Access Dieser Artikel unterliegt den Bedingungen der Creative Commons Attribution License. Dadurch sind die Nutzung, Verteilung und Reproduktion erlaubt, sofern der/die Originalautor/en und die Quelle angegeben sind.

\section{Literatur}

1. Richardson PG. A phase 2 study of bortezomib in relapsed refractory myeloma. New Engl J Med. 2003;348(26):2609-17.

2. Richardson PG. Bortezomib or high-dose dexamethasone for relapsed multiple myeloma. N Engl J Med. 2005;352(24):2487-98.

3. San Miguel J, et al. A practical update on the use of bortezomib in the management of multiple myeloma. Oncologist. 2006;11(1):51-61.

4. Dubois D, et al. Descriptive and prognostic value of patientreported outcomes: the bortezomib experience in relapsed and refractory multiple myeloma. J Clin Oncol. 2006;24(6):976-82.

5. Gulbrandsen N, Hjermstad MJ, Wisloff F. Interpretation of quality of life scores in multiple myeloma by comparison with a reference population and assessment of the clinical importance of score differences. Eur J Haematol. 2004;72(3):172-80.

6. Wisloff F, Gulbrandsen N. Health-related quality of life and patients' perceptions in interferon-treated multiple myeloma patients. Nordic Myeloma Study Group. Acta Oncol. 2000;39(7):809-13.
7. Goldschmidt H. Multiples Myelom. Epidemiologie, ätiologie und pathogenese. Bd. 47 (2). Berlin: Springer Medizin Verlag. 2006.

8. Medicine Io. Crossing the quality chasm: a new health system for the 21st century. Washington: Nat Acad Sci XX; 2001. S. 337.

9. Shaller D. Patient-centered care: what does it take? 2007.

10. Scheibler F, Pfaff H. Shared-Decision Making: Der Patient als Partner im Medizinischen Entscheidungsprozess. Weinheim: Juventa. 2003.

11. Mühlbacher AC, Lincke HJ, Nübling M. Evaluating patients' preferences for multiple myeloma therapy: a discrete-choiceexperiment. Psychosoc Med. 2008;5:10.

12. Mühlbacher A, Nübling M. Analysis of physicians' perspectives versus patients' preferences: direct assessment and discrete choice experiments in the therapy of multiple myeloma. Eur $\mathrm{J}$ Health Econ. 2011;12(3):193-203.

13. Wisloff F, Hjorth M. Health-related quality of life assessed before and during chemotherapy predicts for survival in multiple myeloma. Nordic myeloma study group. Br J Haematol. 1997;97(1):2937.

14. Moeremans K, Annemans L. An update: health economics of managing multiple myeloma. Eur J Cancer. 2006;42(11):168491.

15. Ryan M, Gerard K, Amaya-Amaya M. Using discrete choice experiments to value health and health care: the economics of nonmarket goods and resources. Bd. 11. Dordrecht: Springer; 2008. S. 254.

16. Lancaster KJ. A new approach to consumer theory. J Polit Econ. 1966;74:132-57.

17. McFadden D. Conditional logit analysis of qualitative choice behavior. Zarembka. 1974;1974:105-42.

18. Ben-Akiva ME, Lerman SR. Discrete choice analysis: theory and application to travel demand. MIT Press series in transportation studies. Cambridge: MIT Press; 1985.

19. Backhaus K, et al. Multivariate Analysemethoden: Eine anwendungsorientierte Einführung, Springer-Lehrbuch, Bd 11. Berlin: Springer; 2005. [u.a.]: Springer. VII, 830 S.

20. Bortz J. Statistik für Sozialwissenschaftler. 5., vollst. Überarb. und aktualisierte Aufl, Springer-Lehrbuch. Berlin: Springer; 1999. [u.a.]: Springer. XV, $836 \mathrm{~S}$. 